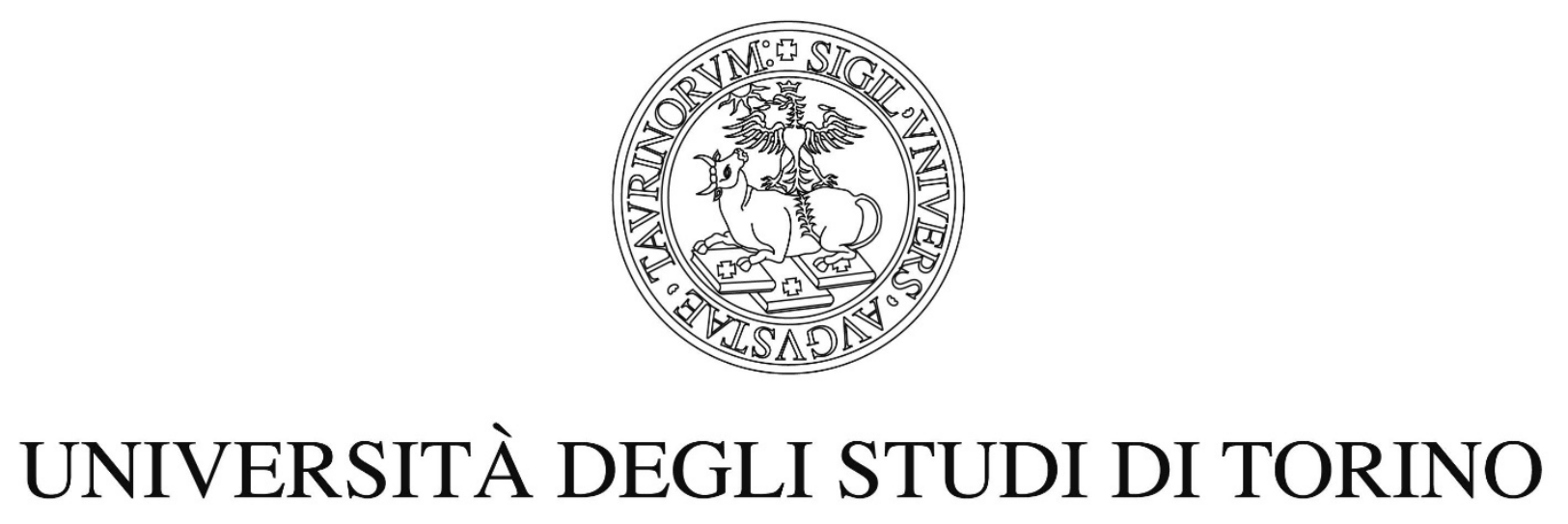

This is an author version of the contribution published on:

Questa è la versione dell'autore dell'opera:

Marco Menin, L'Ambiguïté des larmes :Rousseau et la moralité de l'émotion

\author{
In \\ L'Esprit Créateur, Vol. 52.4/2012 John Hopkins University \\ Press, pp. 107-119
}

DOI: 10.1353/esp.2012.0050

The definitive version is available at:

La versione definitiva è disponibile alla URL:

http://muse.jhu.edu/journals/lesprit_createur/052/52.4.menin.html 


\title{
L'Ambiguïté des larmes : Rousseau et la moralité de l'émotion
}

\author{
Marco Menin
}

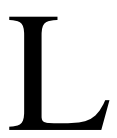

'ÉMOTIVITÉ HUMAINE SE CARACTÉRISE, aux yeux de Rousseau, par une duplicité intrinsèque, dont l'origine doit être recherchée dans l'écart abyssal qui subsiste entre l'homme de la nature et l'homme de l'homme, plus encore que dans les relations mystérieuses et souvent conflictuelles entre l'âme et le corps, longuement illustrées dans la Profession de foi ${ }^{1}$. L'émotion, en fait, s'affirme seulement lors de la sortie de la condition originelle et de l'entrée dans la société civile, qui établit l'accès de l'individu à l'humanité comprise dans le sens le plus élevé (moral et social) du terme ${ }^{2}$. Même si elle représente un fait d'intériorité, une agitation interne qui imprègne tout l'être, l'émotion n'est jamais une question purement autoréférentielle. Elle présuppose de la part du sujet cette reconnaissance de l'altérité (aussi bien à l'égard de l'environnement qu'envers les autres individus) qui est un préliminaire indispensable pour la conquête de la conscience de soi, absente chez l'homme originel. La possibilité de ressentir une émotion implique donc un développement déjà avancé de la dialectique entre les besoins et les désirs qui, à travers la prévalence des seconds sur les premiers, rendue possible par la mémoire et par l'imagination, fait émerger, dans le même temps, les dimensions passionnelles et rationnelles, destinées à se renforcer réciproquement par la suite :

Quoiqu'en disent les Moralistes, l'entendement humain doit beaucoup aux Passions, qui, d'un commun aveu, lui doivent beaucoup aussi : C'est par leur activité, que notre raison se perfectionne ; Nous ne cherchons à connoître, que parce que nous desirons de jouïr, et il n'est pas possible de concevoir pourquoi celui qui n'auroit ni desirs ni craintes se donneroit la peine de raisonner ${ }^{3}$.

Ce lien inséparable entre les besoins physiologiques, passionnels et sociaux-illustré dans la seconde partie du Discours sur l'inégalité-permet de comprendre la vive attention que Rousseau porte aux larmes, qui sont pour lui la manifestation privilégiée et prépondérante de l'émotivité, à partir du témoignage autobiographique : «Peu d'hommes ont autant gémi que moi, peu ont autant versé de pleurs dans leur vie ${ }^{4}$. Il attribue en fait une sorte d' 'exemplarité' aux larmes, non seulement au niveau symbolique mais aussi au niveau anthropologique. Si la fascination esthétique et littéraire que les larmes (objets transparents qui se manifestent en coulant et en disparaissant) durent exercer

(C) L’Esprit Créateur, Vol. 52, No. 4 (2012), pp. 107-119 


\section{L'ESPRIT CRÉATEUR}

sur une conscience qui ne s'arrêta jamais de rechercher une adhésion intuitive et totale au réel, est facilement compréhensible, bien plus complexe, mais en même temps profitable, apparaît leur analyse à la lumière de la question (centrale dans toute l'histoire de la pensée occidentale à partir du Philèbe de Platon) de l'influence des émotions sur la conduite humaine. Dans une telle perspective, en fait, les larmes se révèlent un instrument privilégié pour comprendre plus précisément, d'une part, le lien entre la sensibilité et la moralité qui détermina Rousseau à faire entrer les émotions au cœur de sa réflexion éthique et, d'autre part, pour démontrer l'originalité de sa position par rapport à la vogue contemporaine du sentimentalisme et du 'moralisme larmoyant'.

\section{Larmes physiques / larmes morales}

L'essence suspecte des larmes reflète la genèse complexe de l'émotion, inévitablement suspendue entre l'immédiateté naturelle et l'artifice culturel, entre le processus physiologique et la norme morale. Cette ambivalence apparaît nettement si l'on essaie de parcourir l' 'histoire' des larmes dans le passage de l'état de nature à la société civile. L'homme naturel, qui n'éprouve aucune émotion ne dépassant pas la nécessité de conservation physique, ne pleure pas ; autrement dit, ses larmes ne sont pas de vraies larmes. Dans l'ensemble de son œuvre, Rousseau semble en fait accepter et utiliser de manière implicite la distinction entre les larmes somatiques et les larmes émotives, tout en ne faisant pas toujours la différence de manière nette, au niveau sémantique, entre 'pleurs' et 'larmes' ${ }^{5}$. Les premiers représentent un simple produit physiologique, dû à la réaction mécanique du corps à une stimulation externe ; il s'agit des larmes du 'bon sauvage' qui, à cause de son incapacité à prévoir l'avenir, se trouve privé de grabat où dormir (Discours, OC 3:144) ou de celles du nouveau-né (lequel vit à son tour dans une condition prémorale), qui expriment des besoins vitaux : « En naissant un enfant crie ; sa prémiére enfance se passe à pleurer » (Émile, OC 4:261 $)^{6}$.

Les secondes sont les larmes proprement dites ; celles-ci, même si elles dépendent inévitablement de l'organisation physiologique, sont essentiellement l'expression de la dimension spirituelle qui, par elles, se manifeste à l'extérieur. En ce sens, elles doivent être considérées comme une qualité humaine spécifique, qui marque une distinction importante entre la phase prémorale et l'accession de l'individu à la pleine humanité : «De ces pleurs qu'on croiroit si peu dignes d'attention, nait le prémier raport de l'homme à tout ce qui l'environne : ici se forge le prémier anneau de cette longue chaine dont l'ordre social est formé » (OC 4:286). Contrairement aux gémissements de l'homme originel ou de l'enfant, dont l'origine est à rechercher de manière 


\section{Marco Menin}

exclusive dans le principe d'autoconservation, les racines des larmes 'morales' puisent dans le sentiment, et donc dans « ce qui est proprement humain dans la sensibilité $»^{7}$.

Cette distinction entre larmes physiques et larmes morales-en d'autres termes, larmes somatiques et larmes émotives-renvoie, en dernière instance, à la nature ambivalente de la sensibilité, clairement illustrée dans le second Dialogue. A côté d' « une sensibilité physique et organique qui, purement passive, paroit n'avoir pour fin que la conservation de notre corps », Rousseau distingue une autre sensibilité, « active et morale qui n'est autre chose que la faculté d'attacher nos affections à des êtres qui nous sont étrangers $»^{8}$. Ces deux aspects de la même faculté renvoient respectivement à la sensation, qui unit l'homme aux autres animaux, et au sentiment, qui s'affirme seulement grâce au développement de la dimension politique et morale. Il s'agit d'une distinction de genre, et non d'essence, ce qui complique beaucoup l'analyse des larmes et de l'émotion, par rapport au paradigme cartésien, encore extrêmement influent dans la seconde moitié du dix-huitième siècle.

Pour Descartes, en fait, les émotions sont de simples affections, des modifications passives causées dans l'âme par le mouvement des forces mécaniques (les esprits animaux). Leur fonction naturelle, rendue possible par la médiation de la glande pinéale, est d'inciter l'animal à consentir et à contribuer aux actions qui servent à préserver le corps. C'est pour cela que la tristesse et la joie, qui nous avertissent respectivement de tout ce qui est nuisible ou utile à l'autoconservation, sont les émotions fondamentales : composées avec les quatre autres émotions primitives simples (l'émerveillement, l'amour, la haine et le désir), elles sont à l'origine de toutes les manifestations de la sphère émotive. Dans un tel système de 'psychologie physiologique' le développement des larmes ne peut qu'être marginal et, dans tous les cas, exclusivement somatique. Dans un petit article des Passions de l'âme, intitulé De l'origine des larmes, bien que celles-ci soient d'abord définies comme le fruit d'une tristesse «qui est médiocre et accompagnée ou suivie de quelque sentiment d'amour, ou aussi de joie », elles sont ensuite expliquées à l'intérieur de la pure res extensa : «Et, pour bien entendre leur origine, il faut remarquer que, bien qu'il sorte continuellement quantité de vapeurs de toutes les parties de notre corps, il n'y en a toutefois aucune dont il en sorte tant que des yeux, à cause de la grandeur des nerfs optiques et de la multitude de petites artères par où elles y viennent $»^{9}$.

Rousseau renverse complètement cette conception des larmes. Le fait de pleurer, d'abord simple épiphénomène du mécanisme physiologique, devient, pour lui, l'expression de la complexité de la vie intérieure et signe de l'action 


\section{L'ESPRIT CRÉATEUR}

des émotions sur le corps et sur l'âme. Ce mécanisme est illustré, toujours dans les Dialogues, dans une page où Rousseau met en lumière la concordance parfaite qui règne entre les émotions de Jean-Jacques et leur manifestation physiologique :

Les affections auxquelles il a le plus de pente se distinguent même par des signes physiques. Pour peu qu'il soit ému ses yeux se mouillent à l'instant. Cependant jamais la seule douleur ne lui fit verser une larme ; mais tout sentiment tendre et doux ou grand et noble dont la vérité passe à son cœur lui en arrache infailliblement. Il ne sauroit pleurer que d'attendrissement ou d'admiration : la tendresse et la générosité sont les deux seules cordes sensibles par lesquelles on peut vraiment l'affecter. (OC 1:825)

Il s'agit d'un passage extrêmement important, puisqu'il explicite les deux caractéristiques essentielles de la larme 'morale'. Les larmes qui proviennent du sentiment sont surtout immédiates et involontaires, comme peut le constater Julie : «Des pleurs involontaires s'échapent de mes yeux » $(O C 2: 88)^{10}$. Elles ne suivent pas l'émotion ou n'en sont pas simplement l'instrument, comme le voudrait Descartes, elles en représentent l'essence même. C'est pourquoi les larmes n'ont jamais pour origine la douleur physique seule, dont la portée est, au contraire, minimisée aussi bien du point de vue théorique que du point de vue autobiographique ${ }^{11}$, elles proviennent plutôt d'une vibration de la sensibilité : «Je l'ai vu [Jean-Jacques] se passionner de même, et souvent jusqu'aux larmes pour les choses bonnes et belles dont il étoit frappé dans les merveilles de la nature, dans les œuvres des hommes, dans les vertus, dans les talens, dans les beaux arts et généralement dans tout ce qui porte un caractére de force, de grace ou de vérité digne d'émouvoir une ame sensible » (Dialogues, OC 1:803). L' 'illumination de Vincennes' elle-même, qui marque le début de sa spéculation philosophique à travers la vision d' " un autre monde moral $»^{12}$, est un exemple paradigmatique de ce mécanisme : « J'y passe une demie heure dans une telle agitation qu'en me relevant j'apperçus tout le devant de ma veste mouillé de mes larmes sans avoir senti que j'en repandois » $(O C 1: 1135)^{13}$.

La seconde caractéristique des larmes, et il s'agit de nouveau d'un retournement de la position cartésienne, est leur 'altruisme', c'est-à-dire leur éloignement radical de toute instance égoïste. Si pour Descartes l'émotion ne touche l'âme qu'en ce qui concerne son rapport avec le corps, chez Rousseau, elle est affranchie, grâce au sentiment, de la dimension de la nécessité physique (la 'sensation') qui caractérise inévitablement la sensibilité humaine. Ce n'est pas la douleur, en fait, ou quelque stimulation corporelle que ce soit, qui nous pousse à pleurer, mais la tendresse et la générosité ; ce 


\section{Marco Menin}

n'est pas la souffrance propre qui humidifie les yeux, mais la conscience de la souffrance d'autrui. En somme, les larmes ne sont pas le fruit de l'amour de soi, mais de la pitié qui, au moins dans le second Discours, est présentée comme un principe instinctif, antérieur à la raison, qui « nous inspire une répugnance naturelle à voir perir ou souffrir tout être sensible et principalement nos semblables » (OC 3:126). Ce mouvement vague, lié encore à la sensibilité passive, doit toutefois être élevé à un niveau pleinement moral, être ainsi librement réélaboré en termes sentimentaux, jusqu'à devenir un élan conscient vers l'altérité. Cette métamorphose de la pitié en « prémier sentiment rélatif qui touche le cœur humain selon l'ordre de la nature » est décrite dans l'Émile : « Pour devenir sensible et pitoyable il faut que l'enfant sache qu'il y des êtres semblables à lui, qui souffrent ce qu'il a souffert, qui sentent les douleurs qu'il a senties, et d'autres dont il doit avoir l'idée comme pouvant les sentir aussi » $(O C \text { 4:505 })^{14}$. Dans ce cas aussi, ce sont les larmes qui marquent le plein accès à la moralité : en fait, avant d'avoir compris la pitié, le jeune homme est complètement étranger à l' « art d'affecter la tristesse », et n'a pas non plus « feint de pleurer » $(O C 4: 505)$.

\section{Larmes vraies / larmes fausses}

L'introduction de la question de la 'larme feinte', c'est-à-dire de l'émotion simulée, permet de mettre en lumière une autre particularité de la conception des larmes par Rousseau, conception qui se révèle fondamentale pour marquer sa distance par rapport à toute forme de sentimentalisme ingénu. Nous avons vu comment la complexité des larmes peut être reconduite, à un premier niveau, à leur ambivalence, imputable à deux différents types de sensibilité : la sensibilité passive et physique (sensation) et la sensibilité active et morale (sentiment) qui coexistent dans la constitution de l'être humain. La reconnaissance de la catégorie du sentiment comme principe autonome des émotions permet à Rousseau d'attribuer aux larmes un rôle déterminant dans la conduite morale de l'homme, renversant ainsi la perspective cartésienneet plus généralement, stoïque-selon laquelle la force de l'âme serait constituée par la victoire contre les émotions et l'arrêt des mouvements du corps qui les accompagnent. Une telle prise de position s'inscrit toutefois dans un courant de pensée précis, qui va de Pascal aux moralistes français (La Rochefoucauld, Vauvenargues) et anglais (Shaftesbury), et qui ne semble pas suffisante en elle-même pour rendre compte de l'originalité de la pensée de Rousseau.

La complexité des larmes ne se limite pas à l'ambivalence entre larmes somatiques et larmes émotives-destinée à devenir un leitmotiv de toute la 


\section{L'ESPRIT CRÉATEUR}

littérature sentimentale de la seconde partie du dix-huitième siècle-mais implique, à un second niveau, l'ambiguïté des larmes émotives elles-mêmes, qui relève d'une tension typique de l'œuvre de Rousseau, tension interne à la sensibilité morale. Toujours dans les Dialogues, il est fait une distinction précise entre une « sensibilité positive » qui « dérive immédiatement de l'amour de soi » $(O C$ 1:805) et « est une pure affaire de sentiment où la réflexion n'entre pour rien », et une «sensibilité négative », expression directe de cette dégénération de l'amour de soi qu'est l'amour propre : «Aussitot qu'on prend l'habitude de se mesurer avec d'autres, et de se transporter hors de soi pour s'assigner la prémiére et meilleure place, il est impossible de ne pas prendre en aversion tout ce qui nous surpasse, tout ce qui nous rabbaisse, tout ce qui nous comprime, tout ce qui étant quelque chose nous empêche d'être tout » $(O C \text { 1:806 })^{15}$. Il en découle la dichotomie entre les larmes vertueuses, qui conduisent à la connaissance de soi à travers la reconnaissance de notre prochain et la conscience d'une humanité commune, et les larmes immorales qui expriment, au contraire, l'affirmation d'un élan égoïste. Si les premières sont des larmes transparentes, qui jaillissent spontanément et immédiatement du cœur, les secondes n'ont pas une cause réelle dans l'intériorité, et correspondent à une émotion falsifiée par la dénaturation ou, pire, à une émotion purement externe et mimée.

C'est la raison pour laquelle Rousseau n'hésite pas à condamner avec dureté tout usage 'stratégique' que ce soit des larmes ${ }^{16}$, qui transforme cellesci, plus ou moins ouvertement, en un instrument utile d'affirmation de son propre pouvoir sur les autres. Les larmes malicieuses des femmes corrompues entrent dans cette catégorie, ainsi que les larmes capricieuses des enfants éduqués selon le principe erroné de la dépendance des hommes : «Les prémiéres pleurs des enfants sont des priéres : si on n'y prend garde elles deviennent bientôt des ordres ; ils commencent par se faire assister, ils finissent par se faire servir. Ainsi de leur propre foiblesse [...] nait ensuitel'idée de l'empire et de la domination » (Émile, $O C$ 4:287) ${ }^{17}$. À la lumière de cette distinction, il semble possible de comprendre la véhémence avec laquelle Rousseau attaque le théâtre, sur les scènes duquel les comédiens reproduisent les signes extérieurs des émotions sans ne ressentir en réalité aucun sentiment correspondant. La condamnation méprisante des acteurs, qui est au cœur de la Lettre à d'Alembert et qui sera reprise dans La Nouvelle Hélö̈se ${ }^{18}$, peut se résumer par la vanité de leurs larmes. Celles-ci, pour reprendre une image célèbre de Diderot, jaillissent du cerveau plutôt que du cœur ${ }^{19}$, donnant lieu à « une émotion passagére et vaine, qui ne dure pas plus que l'illusion qui l'a produite ; un reste de sentiment naturel étouffé bientot par les passions ; une 


\section{Marco Menin}

pitié stérile, qui se repait de quelques larmes, et n'a jamais produit le moindre acte d'humanité » (OC 5:23).

Rousseau se montre ainsi parfaitement conscient de la façon dont le problème de l'authenticité des manifestations sentimentales rejoint la réflexion esthétique et sociologique sur le théâtre, pour renverser la possibilité même d'attribuer un rôle moral à l'émotion. La constatation que les larmes, témoignage de la présence à soi et aux autres, peuvent se transformer en simple artifice culturel, en code conventionnel à utiliser selon le bon vouloir de chacun, est la confirmation décisive du fait que l'accès de l'individu à l'émotion n'est pas suffisant en lui-même pour en garantir la bonté et la légitimité. Cette prise de conscience justifie la centralité que Rousseau attribue à l'émotion dans sa définition de l'humanité, au point de ne pas hésiter à connoter l'existence même en clé sentimentale ${ }^{20}$; d'autre part, il marque ainsi nettement sa distance par rapport à un 'culte' irrationnel de l'émotion et de l'abandon aveugle, à l' « onanisme sentimental » ${ }^{21}$ qui devint, au contraire, le code caractéristique de toutes les formes d'expression correspondant à une vulgarisation de la philosophie des sentiments moraux (de la comédie larmoyante au roman sentimental $)^{22}$. Parce qu'elle découle de la perfectibilité humaine, l'émotion peut être, en fait, bonne ou mauvaise, comme toutes les expressions de la vie en société. Contrairement à l'innocence de l'homme de la nature, elle n'est pas donnée une fois pour toutes, elle doit être conquise à travers un véritable apprentissage de la sensibilité, basé sur le bon usage des autres facultés : «Exercer les sens n'est pas seulement en faire usage, [...] c'est apprendre, pour ainsi dire, à sentir » (OC 4:380).

La société moderne, comme le constate Saint-Preux lorsqu'il se rend pour la première fois à Paris, symbole par excellence de la dénaturation sociale, ne reconnaît pas les droits de la sensibilité, qui est, au contraire, suffoquée par le triomphe universel du 'paraître' : «Comme tout n'est que vaine apparence et que tout change à chaque instant, je n'ai le tems d'être ému de rien » $(O C$ $2: 245)$. En contrepoint de cette sorte d'aliénation de l'émotion véritable, la dramatique affirmation d'une émotion feinte et falsifiée (totalement similaire à celle du théâtre), contraint le protagoniste de La Nouvelle Héloüse à renoncer dès le début à ses propres sentiments— « Chaque jour en sortant de chez moi j'enferme mes sentiments sous la clef, pour en prendre d'autres qui se prêtent aux frivoles objets qui m'attendent »-jusqu'à renier en quelque sorte sa propre humanité : «Forcé de changer ainsi l'ordre de mes affections morales ; forcé de donner un prix à des chimeres, et d'imposer silence à la nature et à la raison, je vois ainsi défigurer ce divin modele que je porte au dedans de moi » $(O C$ 2:255). 


\section{L'ESPRIT CRÉATEUR}

Le point crucial consistera alors à réussir à 'libérer' l'émotion des faux objets qui l'absorbent, des artifices qui l'instrumentalisent et la corrompent. Cette tâche ne peut être accomplie que par l'émotion elle-même, entendue dans son acception vertueuse, comme le confirme justement l'analyse des larmes. Dans la Lettre à d'Alembert, toujours, Rousseau critique en fait la théorie rhétorique de l'abbé Du Bos, qui voyait entre les émotions réelles et celles qui étaient représentées au théâtre une simple différence de degré ${ }^{23}$. Pour Rousseau, au contraire, il y a une différence de nature qui réside dans l'incapacité de l'émotion récitée d'instaurer une authentique relation morale entre les individus : «L'on croit s'assembler au Spectacle, et c'est-là que chacun s'isole ; c'est-là qu'on va oublier ses amis, ses voisins, ses proches, pour s'intéresser à des fables, pour pleurer les malheurs des morts $»^{24}$. Ce n'est pas un hasard s'il compare les «pleureuses de loges », si fières de leurs larmes, à la cruelle Messaline qui n'hésite pas à s'émouvoir (extérieurement seulement) devant la défense éloquente de Valerius Asiaticus, quitte ensuite à demander à Vitellius de le condamner à mort (OC 5:23, variante a).

Ce type de larmes témoigne en définitive d'une humeur égoïste, et exprime une émotion superficielle limitée à l'exaltation du moment, qui ne se transforme pas en un sentiment durable dans le temps et qui n'est pas en mesure de contribuer d'aucune manière à l'action dans le monde ni de la diriger :

Au fond, quand un homme est allé admirer de belles actions dans des fables, et pleurer des malheurs imaginaires, qu'a-t-on encore à éxiger de lui ? N'est-il pas content de lui-même ? Ne s'applaudit-il pas de sa belle ame ? Ne s'est-il pas acquitté de tout ce qu'il doit à la vertu par l'hommage qu'il vient de lui rendre ? Que voudroit-on qu'il fit de plus ? Qu'il la pratiquât luimême ? Il n'a point de rôle à jouer : il n'est pas Comédien. (OC 5:23-24)

Il y a, en somme, une différence émotive entre les larmes immorales et les larmes vertueuses, que ce soit en termes de profondeur ou d'extension. Si les premières se caractérisent par un pic d'intensité éphémère suivi par un déclin rapide, les secondes se développent et arrivent à maturité dans l'intimité, facilitant la transformation de l'émotion en un sentiment moral qu'il est possible de partager immédiatement. Le « pur sentiment» qui fait couler « les douces larmes » est en fait un sentiment universel, « aussi naturel au cœur humain que l'amour de soi-même » (OC 5:22).

L'insistance de Rousseau sur la divergence entre les larmes vertueuses et les larmes immorales, ainsi que sa volonté de reconduire les secondes exclusivement à la dégénération (historique et accidentelle et non essentielle à la nature humaine), paraît justifiée à la lumière de la fonction directive détermi- 


\section{Marco Menin}

nante qu'il attribue aux émotions dans la conduite humaine. Il est, en fait, possible de soutenir que c'est, précisément, l'ambiguïté des vraies larmes, laquelle conserve l'innocence naturelle tout en l'élevant à la moralité civique, qui sauve l'émotivité du discrédit dans lequel semble la jeter, d'emblée, la possibilité de la simulation. La critique la plus fréquente à l'égard de toute théorie philosophique qui prétend fonder sur les émotions une position morale consiste justement à insinuer le doute sur le caractère circonstanciel et conventionnel de l'émotion ${ }^{25}$. En dernière instance, celle-ci resterait toujours liée à une seule éventualité, ne permettant pas la construction d'un modèle moral susceptible d'être généralisé. Dans cette perspective, l'émotion peut tout au plus être envisagée comme une fonction morale subordonnée et provisoire, substitut, sensible et biologique, de la raison. Cette objection tombe, ou pour le moins, perd une grande partie de sa prégnance, dans la conception de la sensibilité de Rousseau qui, en nouant inséparablement sensation et sentiment, organisation physiologique et ordre moral, permet d'affirmer à la fois, et sans contradiction, l'occurrence isolée de l'émotion (inévitablement liée à son ambivalence) et sa validité universelle (qui découle au contraire de l'ambigüité de la sensibilité active et morale, comprise dans son aspect positif).

L'émotion devient ainsi l'essence même d'une relation authentiquement morale entre les individus, puisqu'elle permet de manifester à l'extérieur sa propre intériorité : «Rien ne lie tant les cœurs que la douceur de pleurer ensemble » $(O C$ 1:529). Dans cette perspective, on doit considérer les larmes comme la forme la plus efficace de communication, dont la sincérité s'oppose à la fragilité des paroles souvent mensongères. De manière significative, Rousseau impute la naissance même du langage non aux besoins physiques, comme le voulaient les sensualistes, mais aux passions et aux besoins moraux. Pour cette raison, le « langage des prémiers hommes », basé sur l'expression corporelle des sentiments, ne fut pas « langu[e] de Geométres », mais «langu[e] de Poëtes » (Essai, OC 5:380) : «Les premiéres voix [de l'homme] sont la plainte et les pleures » (Émile, manuscrit Favre, OC 4:76).

La supériorité de ce langage naturel, perdu en grande partie durant le processus historique de dénaturation, trouve de nombreuses confirmations aussi bien dans les écrits autobiographiques que dans l'œuvre littéraire. Dans ses rapports avec Mme d'Houdetot, par exemple, les larmes seules sont capables d'exprimer vraiment l'intériorité de Jean-Jacques amoureux : «Je trouvai pour rendre les mouvemens de mon cœur un langage vraiment digne d'eux. Ce fut la prémiére et l'unique fois de ma vie [...]. Que d'enivrantes larmes je versai sur ses genoux ! que je lui en fis verser malgré elle !» (Confessions, $O C 1: 444)^{26}$. Il en est de même pour Émile et Sophie, qui «versent 


\section{L'ESPRIT CRÉATEUR}

quelquefois ensemble des larmes plus pures que la rosée du ciel, et ces douces larmes font l'enchantement de leur vie » (OC 4:792), ainsi que pour SaintPreux et Julie, dont la réciprocité sentimentale est tellement marquée que le jeune homme peut observer que « [s]on cœur est inondé des pleurs qui coulent de tes yeux » $(O C$ 2:338).

Au-delà des nuances, différentes pour chaque cas, l'analyse des larmes morales semble démontrer avec force comment la théorie des émotions, chez Rousseau, est totalement centrée sur le caractère désintéressé de ces dernières, qui garantit à la fois leur valeur universelle et leur infaillible droiture morale, ressentie immédiatement comme telle sans aucune référence à un quelconque paramètre extérieur. La manifestation d'une émotion n'a, en définitive, rien à voir avec son objectivation, elle « possède le caractère ambigu et presque contradictoire d'une épiphanie [...]. En se dévoilant, l'intériorité ne se détache pas de ses origines. Elle ne rejoint pas les objets ne serait-ce qu'en l'espèce de signes. Elle ne s'inscrit pas dans le monde à la façon d'un texte qui serait disponible pour tout ${ }^{27}$. Cette sorte de paradoxe trouve chez Rousseau sa représentation la plus efficace dans les larmes, dont l'ambiguïté fondamentale réside - en dernière instance_-dans le fait qu'elles n'appartiennent pas complètement à la personne individuelle, même si, en quelque sorte, dans leur coappartenance solidaire et conflictuelle à l'âme et au corps, elles en manifestent la complexité et le mystère.

C'est alors que jaillit l'extraordinaire capacité des larmes à transformer l'irréversible en réversible, à entamer l'inéluctabilité du mal qui caractérise l'existence humaine, lui faisant perdre son opacité et son obscurité, jusqu'à l'illuminer progressivement et le transformer en joie, à travers un authentique processus de libération morale décrit avec pénétration dans Émile et Sophie : «Au lieu de la séche douleur qui me consumoit auparavant, j'avois la douceur de m'attendrir jusqu' aux larmes » (OC 4:898). Les larmes deviennent ainsi une contrepartie inséparable du bonheur ${ }^{28}$, une forme de pacification intérieure que tous les hommes peuvent atteindre. Même l'athée Wolmar, qui ne connaît pas le vrai sentiment et qui n'hésite pas à se définir « homme sans passion », reçoit de manière inattendue le don des larmes grâce à l'amour de Julie : «Ces mots prononcés avec tendresse m’émurent au point qu'en portant fréquemment à ma bouche ses mains que je tenois dans les miennes, je les sentis se mouiller de mes pleurs. Je ne croyois pas mes yeux faits pour en répandre. Ce furent les premiers depuis ma naissance ; ce seront les derniers jusqu'à ma mort » $(O C \text { 2:429 et 721 })^{29}$.

À l'opposé des fausses émotions, qui peuvent tout au plus conduire à un plaisir sporadique, les vraies larmes, dont il n'est jamais possible de disposer, 


\section{Marco MENin}

mais qui peuvent seulement être offertes et reçues, permettent à l'individu d'accéder à une forme d'élévation morale qui, comme avait précisément observé La Fontaine, élève l'individu au-delà de sa condition humaine pour le rapprocher de la condition divine : «Les mortels sont mortels quand ils pleurent de leurs douleurs ; mais, quand ils pleurent des douleurs d'autrui, ce sont proprement des dieux $»^{30}$.

\section{Université de Turin, Italie}

\section{Notes}

1. «Unie à un corps mortel par des liens non moins puissans qu'incompréhensibles, le soin de la conservation de ce corps excite l'ame à rapporter tout à lui, et lui donne un intérest contraire à l'ordre général », Jean-Jacques Rousseau, Émile ou de l'éducation, Euvres complètes, vol. 4, Bernard Gagnebin et Marcel Raymond, éds. (Paris: Gallimard, 1969), 603.

2. «Ce passage de l'état de nature à l'état civil produit dans l'homme un changement très rémarquable, en substituant dans sa conduite la justice à l'instinct, et donnant à ses actions la moralité qui leur manquoit auparavant », Du Contrat social, Euvres complètes, vol. 3, Bernard Gagnebin et Marcel Raymond, éds. (Paris: Gallimard, 1964), 364.

3. Jean-Jacques Rousseau, Discours sur l'origine de l'inégalité, OC 3:143.

4. Jean-Jacques Rousseau, Les Confessions, Euvres complètes, vol. 1, Bernard Gagnebin et Marcel Raymond, éds. (Paris: Gallimard, 1959), 103.

5. Cette distinction avait été approfondie et longuement décrite, dans la seconde partie du dixseptième siècle, par le médecin Cureau de la Chambre, qui en fit la base de son extrêmement complexe classification des larmes : «Ainsi toutes les Larmes sont des Pleurs, mais tous les Pleurs ne sont pas des Larmes », Marin Cureau de la Chambre, Les Charactères des passions : dernier volume (Amsterdam: Antoine Michel, 1663), 14. Sur la conception des larmes au dix-septième siècle voir Sheila Page Bayne, Tears and Weeping : An Aspect of Emotional Climate Reflected in Seventeenth-Century French Literature (Tübingen: Gunter Narr Verlag, 1981), en particulier 85-90.

6. Le lien entre cette forme de larme et les besoins physiologiques est confirmé dans la suite : «L'enfant sent ses besoins, et ne les peut satisfaire, il implore le secours d'autrui par des cris » $(O C$ 4:286).

7. Jean Sgard, entrée «Sentiment », in Dictionnaire de Jean-Jacques Rousseau, Raymond Trousson et Frédéric Eigeldinger, éds. (Paris: Champion, 1996), 854. Pour une analyse détaillée de ces thématiques voir Le Vocabulaire du sentiment dans l'œuvre de JeanJacques Rousseau, Michel Gilot et Jean Sgard, éds. (Genève: Slatkine, 1980), 6-11.

8. Jean-Jacques Rousseau, Rousseau juge de Jean-Jacques : dialogues, OC 1:805

9. René Descartes, Les Passions de l'âme, Euvres complètes, Charles Adam et Paul Tannery, éds. (Paris: Vrin, 1964-1974), 11:754. Sur la théorie cartésienne des larmes voir Ann Wilbur Mackenzie, « Descartes on Life and Sense », Canadian Journal of Philosophy, 19 (1989): 163-92 ; Tom Lutz, Crying : The Cultural and Natural History of Tears (New York: W.W. Norton, 1999), 74-76.

10. La même expérience est décrite par Claire : «Je me suis sentie en pleurs sans savoir pourquoi » $(O C$ 2:620).

11. «Le mal moral est incontestablement nôtre ouvrage, et le mal physique ne seroit rien sans nos vices qui nous l'ont rendu sensible » (Émile, OC 4:587). L'indifférence pour le mal physique et l'extraordinaire sensibilité envers le mal moral sont présents chez Jean-Jacques dès son enfance, comme le confirme sa réaction quand il est injustement puni pour avoir cassé les dents d'un peigne (OC 1:20).

12. Jean-Jacques Rousseau, Les Rêveries du promeneur solitaire, OC 1:1015. 


\section{L'ESPRIT CRÉATEUR}

13. L'expérience de l'illumination de Vincennes est racontée en divers lieux et à chaque fois l'analogie entre le bouleversement moral et le bouleversement physique se représente (OC $1: 351,828-29$ et 1041).

14. Le rôle de l'imagination est bien clarifié dans l'Essai sur l'origine des langues : « La pitié, bien que naturelle au cœur de l'homme resteroit éternellement inactive sans l'imagination qui la met en jeu. Comment nous laissons-nous émouvoir à la pitié ? En nous transportant hors de nous-mêmes ; en nous identifiant avec l'être souffrant », CEuvres complètes, vol. 5, Bernard Gagnebin et Marcel Raymond, éds. (Paris: Gallimard, 1995), 395. Voir Paul Audi, "Sur le rôle de l'imagination dans l'exercice de la pitié », Rousseau : une philosophie de l'âme (Paris: Verdier, 2007), 421-33.

15. Dans l'Émile aussi « les passions repoussantes et cruelles, qui rendent, pour ainsi dire, la sensibilité non seulement nulle, mais négative » sont condamnées (OC 4:506).

16. Voir Jean-Jacques Roubine, "La Stratégie des larmes au XVII ${ }^{\mathrm{e}}$ siècle », Littérature, 9 (1973): 56-73.

17. Ces larmes devront être ignorées par le pédagogue : «Dés qu'il [l'enfant] peut demander en parlant ce qu'il desire, et que pour l'obtenir plus vîte ou pour vaincre un refus il appuye de pleurs sa demande, elle lui doit être irrévocablement refusée » (OC 4:312).

18. Même le mendiant est, aux yeux de Julie, moins inutile qu'un acteur : « Pourquoi ne récompenserois-je pas l'éloquence de ce mendiant qui me remue le cœur et me porte à le secourir, comme je paye un Comédien qui me fait verser quelques larmes stériles? Si l'un me fait aimer les bonnes actions d'autrui, l'autre me porte à en faire moi-même : tout ce qu'on sent à la tragédie s'oublie à l'instant qu'on en sort ; mais la mémoire des malheureux qu'on a soulagés donne un plaisir qui renait sans cesse » (OC 2:539). Sur le personnage de l'acteur dans la pensée de Rousseau voir Jean Rousset, "Qu'est-ce que le talent du comédien ? », Annales Jean-Jacques Rousseau, 37 (1966-1968): 19-34.

19. «Les larmes du comédien descendent de son cerveau ; celles de l'homme sensible montent de son cœur ", Denis Diderot, Paradoxe sur le comédien, Euvres complètes, Herbert Dieckmann, Jean Varloot et Jacques Proust, éds. (Paris: Hermann, 1975-2004), 20:57. Diderot est toutefois sincèrement convaincu de la vertu moralisatrice du théâtre : "Quelquefois j'ai pensé qu'on discuterait au théâtre les points de morale les plus importants », De la poésie dramatique, OC 10: 419.

20. «Exister pour nous, c'est sentir ; notre sensibilité est incontestablement antérieure à nôtre intelligence, et nous avons eu des sentimens avant des idées » (OC 4:600).

21. André Monglond, Le Préromantisme français, (Paris: Corti, 1965), 2:318.

22. Voir William M. Reddy, The Navigation of Feeling : A Framework for the History of Emotions (Cambridge: Cambridge U P, 2001), 161-72 ; Anne Coudreuse, Le Goût des larmes au XVIII ${ }^{e}$ siècle (Paris: P U F, 1999), 55-125.

23. «Les Peintres \& les Poëtes excitent en nous ces passions artificielles, en présentant les imitations des objets capables d'exciter en nous des passions véritables. Comme l'impression que ces imitations font sur nous est du même genre que l'impression que l'objet imité par le Peintre ou par le Poëte feroit sur nous : comme l'impression que l'imitation fait n'est différente de l'impression que l'objet imité feroit, qu'en ce qu'elle est moins forte, elle doit exciter dans notre ame une passion qui ressemble à celle que l'objet imité y auroit pû exciter », Jean-Baptiste Du Bos, Réflexions critiques sur la poésie et sur la peinture (Utrecht: Chez Étienne Neaulme, 1732), 15.

24. Lettre à d'Alembert sur les spectacles, OC 5:16.

25. Voir Robert C. Solomon, "In Defense of Sentimentality », Philosophy and Literature, 14 (1990): 304-23, et In Defense of Sentimentality : The Passionate Life (Oxford: Oxford U P, 2004).

26. Sur l'éloquence des larmes au dix-huitième siècle voir Anne Vincent-Buffault, Histoire des larmes : XVIII ${ }^{e}$-XIX ${ }^{e}$ siècles (Paris: Rivage, 1986), 24-45 ; « Le Langage des larmes aux siècles classiques », Adélaïde Cron et Cécile Lignereux, éds., Littératures classiques, 62 (2007) ; pour une étude plus générale voir Jean-Loup Charvet, L'Éloquence des larmes (Paris: Desclée De Brouwer, 2000).

27. Luc Boltanski, La Souffrance à distance : morale humanitaire, médias et politique (Paris: Métaillé 1993), 124. 


\section{Marco Menin}

28. «L'excessive joye elle-même arrache des pleurs plutôt que des ris »; « On avoit le sourire à la bouche et les yeux en pleurs » (OC 2:238 et 738).

29. Pour la conception des larmes dans La Nouvelle Héloüse voir Siegfried Jüttner, « Weinende Herzen: die Natur der Tränen in der Nouvelle Hélö̈se von Rousseau », in Das Weinende Saeculum, K. Bartèolke, éd. (Heidelberg: C. Winter, 1983), 49-60 ; pour une interprétation philosophique des larmes de Wolmar, voir Franck Salaün, « Les Larmes de Wolmar : Rousseau et le problème du matérialisme », in Rousseau et la philosophie, André Charrak et Jean Salem, éds. (Paris: Publications de la Sorbonne, 2004), 71-86.

30. Jean de La Fontaine, Les Amours de Psyché et de Cupidon, Euvres complètes, Jean Marmier, éd. (Paris: Seuil, 1965), 425. 